\title{
DEMANDAS PSICOLÓGICAS, CONTROLE E APOIO SOCIAL NO TRABALHO DE AGENTES COMUNITÁRIOS DE SAÚDE*
}

\author{
Gilmara Aparecida Batista Fernandes ${ }^{1}$, Angélica da Conceição Oliveira Coelho², Heloisa Campos Paschoalin², \\ Leila Maria Mansano Sarquis ${ }^{3}$, Rosângela Maria Greco ${ }^{4}$
}

\begin{abstract}
RESUMO: Objetivo: identificar as demandas psicológicas, o controle e o apoio social no trabalho dos Agentes Comunitários de Saúde. Método: estudo descritivo, com abordagem quantitativa, realizado com 212 trabalhadores da Atenção Primária à Saúde de um município do interior de Minas Gerais. Os dados foram coletados entre julho e outubro de 2015 com Agentes Comunitários de Saúde das Unidades de Atenção Primária de Saúde. A análise foi descritiva com frequências absolutas e relativas. Como variáveis independentes características sociodemográficas e do trabalho, e como variáveis dependentes a escala reduzida Modelo Demanda-Controle e o apoio social no trabalho. Resultados: evidencia-se uma população feminina em fase produtiva da vida, o trabalho passivo e alta exigência tiveram maiores proporções, demonstrando alto risco de desenvolvimento do estresse psicossocial no trabalho. Conclusão: ressalta-se a necessidade de intervenções nas condições de vida e laborais, principalmente no que se refere a aspectos psicossociais e organizacionais.

DESCRITORES: Saúde do trabalhador; Trabalho; Condições de trabalho; Atenção primária à saúde; Agentes comunitários de saúde.

\section{PSYCHOLOGICAL DEMANDS, CONTROL AND SOCIAL SUPPORT IN THE WORK OF COMMUNITY HEALTH AGENTS}

\begin{abstract}
Objective: To identify the psychological demands, control and social support in the work of Community Health Agents Method: Descriptive study, with a quantitative approach, carried out with 212 primary health care workers from a municipality in the interior of Minas Gerais. Data was collected between July and October 2015 from Community Health Agents of Primary Health Care units. Sociodemographic and labor characteristics were the independent variables and short scale Demand-Control Model and social support at work were the dependent variables. Results: Female working-age population. Passive job and high demand were prevalent, indicating high risk of development of psychosocial stress at work. Conclusion: Interventions in living and working conditions are necessary, especially regarding psychosocial and organizational aspects.
\end{abstract}

DESCRIPTORS: Worker's health; Job; Working conditions; Primary health care; Community health agents.

\section{DEMANDAS PSICOLÓGICAS, CONTROL Y APOYO SOCIAL EN EL TRABAJO DE AGENTES COMUNITARIOS DE SALUD}

RESUMEN: Objetivo: identificar las demandas psicológicas, el control y el apoyo social en el trabajo de los Agentes Comunitarios de Salud. Método: estudio descriptivo, con abordaje cuantitativo, que se realizó con 212 trabajadores de la Atención Básica a la Salud de un municipio del interior de Minas Gerais. Se recogieron los datos entre julio y octubre de 2015 con Agentes Comunitarios de Salud de las Unidades de Atención Básica de Salud. El análisis usado fue el descriptivo con frecuencias absolutas y relativas. Se utilizaron como variables independientes las características social, demográficas y del trabajo, y como variables dependientes la escala reducida Modelo Demanda-Control y el apoyo social en el trabajo. Resultados: se evidencia una población femenina en etapa productiva de la vida; el trabajo pasivo y la gran exigencia presentaron mayores proporciones, demostrando alto riesgo de desarrollo de estrese psicosocial en el trabajo.

DESCRIPTORES: Salud del trabajador; Trabajo; Condiciones de trabajo; Atención básica a la salud; Agentes comunitarios de salud. *Artigo extraído da dissertação de mestrado "Demandas Psicológicas, Controle e Apoio Social no Trabalho de Agentes
Comunitários de Saúde". Universidade Federal de Juiz de Fora, 2016.

${ }^{1}$ Enfermeira. Mestre em Enfermagem. Universidade Federal de Juiz de Fora. Juiz de Fora, MG, Brasil.

Enfermeira. Doutora em Enfermagem. Docente de Enfermagem da Universidade Federal de Juiz de Fora. Juiz de Fora, MG, Brasil.

${ }^{3}$ Enfermeira. Doutora em Enfermagem. Docente de Enfermagem da Universidade Federal do Paraná. Curitiba - PR - Brasil. ${ }^{4}$ Enfermeira. Doutora em Saúde Pública. Docente de Enfermagem da Universidade Federal de Juiz de Fora. Juiz de Fora, MG, Brasil. 


\section{INTRODUÇÃO}

O trabalho tem papel fundamental em todos os âmbitos da vida do ser humano, determina a formação de identidades e possui relevância em uma abordagem que considera os aspectos subjetivos do trabalhador e a sua participação social. É transformador, e favorece ao indivíduo entender e potencializar a dimensão da subjetividade, não somente produzir, mas transformar a si mesmo. ${ }^{(1)}$

Há, portanto, uma relação entre os indivíduos e o trabalho para a manutenção de suas necessidades na busca pela qualidade de vida e saúde. Porém, devido às rápidas transformações tecnológicas, os trabalhadores têm ficado vulneráveis, sofrido influências de fatores psicossociais paradoxais e consequente exposição de prejuízos à saúde.(2)

Os riscos ocupacionais integram o processo de trabalho e se relacionam com os elementos laborais, o que pode produzir sobrecarga psíquica nos indivíduos. Destacam-se os riscos psicossociais, pois são complexos e envolvem características relacionadas ao indivíduo (personalidade), ao ambiente de trabalho (demandas e controle sobre a tarefa) e ao ambiente social (fatores culturais). ${ }^{(3-5)}$

No que se refere à saúde dos trabalhadores da atenção primária, destacam-se a complexidade de assistência e as diversas exigências que incidem sobre estes trabalhadores, são elas: deficiências do sistema de saúde, más condições de trabalho, com exposição a cargas físicas e psicossociais elevadas e a situações de pobreza e desigualdades, entre outras. ${ }^{(3)} \mathrm{O}$ cenário da Atenção Primária à Saúde (APS), principalmente a Estratégia de Saúde da Família (ESF), apresenta riscos psicossociais, gerados principalmente pela organização do trabalho, que podem gerar estresse ocupacional(6).

Desta forma, os profissionais da ESF, especialmente os Agentes Comunitários de Saúde (ACS), ao visarem a promoção da saúde da comunidade por meio da prevenção de doenças, através de trabalho coletivo que pressupõe relações interpessoais entre a equipe e a comunidade, podem estar expostos a estressores psicossociais ${ }^{(6-7)}$.

A motivação em realizar este estudo advém da importância de avaliar as condições de trabalho e saúde dos profissionais da APS, em especial dos ACS, tendo em vista que esses trabalhadores possuem papel articulador e facilitador para o desenvolvimento do vínculo entre a comunidade e a equipe de saúde.

Dessa forma, este estudo objetivou identificar as demandas psicológicas, o controle e o apoio social no trabalho dos Agentes Comunitários de Saúde. Tais fatores, quando em desequilíbrio, podem favorecer ao aumento de adoecimento e desenvolvimento do estresse psicossocial no trabalho.

\section{MÉTODO}

Estudo quantitativo, descritivo e exploratório, de corte transversal, desenvolvido com Agentes Comunitários de Saúde (ACS) de 42 Unidades de Atenção Primária de Saúde (UAPS) do município de Juiz de Fora, que possui meio milhão de habitantes aproximadamente e fica localizado na microrregião da Zona da Mata do Estado de Minas de Gerais (MG).

A população do estudo foi composta por amostra probabilística aleatória simples, com 212 participantes. Para tal, foi considerado o intervalo de confiança de 95\% (IC 95\%), margem de erro ponderado de acordo com a população de $6 \%$, prevalência de $50 \%$ (dada à multiplicidade de desfechos analisados e ausência prévia de estudo com essa perspectiva), e possíveis perdas por recusa de aproximadamente $25 \%$.

Os critérios de inclusão foram ser ACS e estar em exercício de sua profissão. Foram excluídos os trabalhadores que não foram encontrados durante três tentativas de contato por telefone ou em visita do pesquisador à UAPS durante a coleta de dados.

A coleta de dados foi realizada no período de julho a outubro de 2015 no ambiente de trabalho dos ACS, por meio de questões referentes às variáveis independentes sociodemográficas (sexo, idade, estado civil e grau de instrução), laborais (turno, carga horária, outro emprego e tempo no cargo) 
e aplicação de um questionário com foco na organização do trabalho com a escala originalmente elaborada por Karasek, e reduzida por Tores Theorell em 17 questões, considerando as três dimensões (demanda, controle e apoio social), denominada Job Stress Scale ou Escala Sueca de Demanda Controle Apoio social (DCS). Utilizou-se a versão brasileira traduzida e adaptada para o português por Alves (2004) e considerada como Modelo Demanda-Controle (MDC). ${ }^{(8-9)}$

Os quadrantes do MDC de Karasek foram construídos com base na mediana da distribuição e dicotomizados em dimensões de alta e baixa demanda, controle, apoio social. As opções de respostas para as dimensões "demanda psicológica" e "controle" foram variações entre "frequentemente" e "nunca/quase nunca" e para a dimensão "apoio social" entre "concordo totalmente" e "discordo totalmente", apresentadas em escala do tipo Likert (1-4). ${ }^{(9)}$

A cada resposta foi atribuído um escore de 1 a 4, considerando-se itens com pontuação reversa nas duas primeiras dimensões. Os escores foram obtidos pela soma dos itens de cada dimensão e variaram de 5-20 (demanda) com pontuação entre 5 e 14 baixa demanda e valores acima de 14 alta demanda, 6-24 (controle) classificando-se como baixo controle pontuação entre 5 e 17 e acima de 17 como alto controle e para a dimensão apoio social, o ponto de corte até 17 para baixo apoio social e, acima de 17 pontos, para alto apoio social. ${ }^{(9)}$

A demanda psicológica pode ser definida pela percepção do indivíduo em relação à intensidade com que lhe é exigida a realização de tarefas, ou seja, à sobrecarga de trabalho, já o controle no trabalho é a capacidade do trabalhador de decidir sobre as relações e conflitos de trabalho, desenvolvimento da tomada de decisão e de habilidades, e a dimensão do apoio social é considerada como a integração social e auxilia na realização das tarefas laborais, atuando como moderadora de efeitos de desgaste sobre a saúde do trabalhador. ${ }^{(8)}$

Diante dessa classificação, torna-se possível realizar quatro combinações: trabalho com alta exigência (alta demanda e baixo controle), que é o tipo de trabalho altamente estressante, trabalho passivo (baixa demanda e baixo controle), baixa exigência (baixa demanda e alto controle), e trabalho ativo (alta demanda e alto controle). ${ }^{(8)}$

A combinação mais prejudicial para a saúde dos trabalhadores é o trabalho com alta exigência, por ser fonte de estresse devido ao desgaste gerado, podendo ter como consequência o adoecimento físico e psicológico. Já o trabalho com baixa exigência é considerado o trabalho desejável, pois se configura em um estado altamente confortável e ideal de trabalho, possibilitando relaxamento e menor chance de situações estressantes. ${ }^{(8-10)}$

Portanto, a avaliação do risco para o estresse no trabalho é realizada a partir da combinação de níveis altos e/ou baixos de interação de demandas psicológicas, de controle e apoio social, os quais provocam riscos diferenciados à saúde do trabalhador. ${ }^{(8-10)}$

Para análise dos dados utilizou-se o programa Statistical Package for the Social Sciences (SPSS). Foi realizada análise descritiva por meio de frequências absolutas e relativas e análise do Modelo Demanda-Controle-Apoio social no trabalho.

A pesquisa foi aprovada pelo Comitê de Ética em Pesquisas com Seres Humanos da UFJF, com parecer de $n^{\circ} 932.706$ e em conformidade com as orientações da Resolução 466/12 do Conselho Nacional de Saúde do Ministério da Saúde. ${ }^{(11)}$

\section{- RESULTADOS}

Ao analisar os aspectos sociodemográficos, houve predominância do sexo feminino 193 (91,5\%), idade média de 44 anos com desvio padrão de 9,93, idade mínima de 23 anos e máxima de 71 anos. Em relação à situação conjugal, 117 (55,5\%) eram casados ou em união estável. A maioria dos ACSs 114 (54\%) possui ensino médio completo e $45(21,3 \%)$ relataram ter ensino técnico (Tabela 1$).$ 
Tabela 1 - Frequência das características sociodemográficas dos Agentes Comunitários de Saúde. Juiz de Fora, MG, Brasil, 2016

\begin{tabular}{lcc} 
Características sociodemográficas & n & $\%$ \\
\hline Idade & & \\
\hline Mínima & 23 & - \\
\hline Máxima & 71 & - \\
\hline 20 a 36 anos & 58 & 27,5 \\
\hline 37 a 45 anos & 60 & 28,4 \\
\hline Acima de 46 anos & 93 & 44,1 \\
\hline Sexo & & \\
\hline Masculino & 18 & 8,5 \\
\hline Feminino & 193 & 91,5 \\
\hline Cor/Raça* & & \\
\hline Preta & 36 & 17,1 \\
\hline Parda & 72 & 34,1 \\
\hline Branca & 100 & 47,4 \\
\hline Amarela & 3 & 1,4 \\
\hline Estado Conjugal & & \\
\hline Casado/União Estável & 117 & 55,5 \\
\hline Não casados & 94 & 44,5 \\
\hline Escolaridade & & \\
\hline Até o Ensino Médio incompleto & 13 \\
\hline Ensino Médio Completo & 6,2 \\
\hline Ensino Técnico & 114 & 54 \\
\hline Universitário ou mais & 45 & 21,3 \\
\hline Total & 39 & 18,5 \\
\hline & 212 & 100 \\
\hline & & \\
\hline & & \\
\hline
\end{tabular}

Em relação às características laborais dos ACS, 180 (84,9\%) têm carga horária semanal de 40h, e 188 $(89,1 \%)$ referiram ter começado a trabalhar antes dos 20 anos de idade. Quanto ao tempo de trabalho na APS 127 (59,9\%) relataram ter entre 11 a 20 anos e $85(40,1 \%)$ ter menos de dez anos de trabalho na APS (Tabela 2).

Tabela 2 - Frequência das características individuais relacionadas ao trabalho dos Agentes Comunitários de Saúde. Juiz de Fora, MG, Brasil, 2016 (continua)

\begin{tabular}{lcc} 
Características relacionadas ao trabalho & $\mathbf{n}$ & $\%$ \\
\hline Carga horária (semanal) & 180 & 84,9 \\
\hline 40 horas & 32 & 15,1 \\
\hline$>40$ horas & \\
\hline Idade em que começou a trabalhar & 188 & 89,1 \\
\hline$<20$ anos & 23 & 10,9 \\
\hline Acima de 20 anos & \\
\hline Trabalha no período noturno & 24 & 11,3 \\
\hline Sim & 188 & 88,7 \\
\hline Não & \\
\hline Número de empregos & 188 & 88,3 \\
\hline Um emprego & 24 & 11,3 \\
\hline$\geq 2$ empregos
\end{tabular}




\begin{tabular}{lcc}
\hline Tempo de serviço na APS & & \\
\hline Menos de 5 anos & 60 & 28,3 \\
\hline De 6 a 10 anos & 25 & 11,8 \\
\hline De 11 a 15 anos & 101 & 47,6 \\
\hline De 16 a 20 anos & 26 & 12,3 \\
\hline Total & 212 & 100
\end{tabular}

No que diz respeito à demanda, ao controle e ao apoio social no trabalho dos ACSs, 114 (53,8\%) apresentaram alta demanda, $138(65,1 \%)$ baixo controle e a maior proporção dos trabalhadores 140 $(66,4 \%)$ possuem alto apoio social (Tabela 3$)$.

Tabela 3 - Caracterização das Demandas psicológicas, Controle e Apoio Social no trabalho dos Agentes Comunitários de Saúde. Juiz de Fora, MG, Brasil, 2016

\begin{tabular}{lcc} 
Caracterização Modelo Demanda-Controle & N* & $\%$ \\
\hline Demanda Psicológica & & \\
\hline Baixa Demanda & 98 & 46,2 \\
\hline Alta Demanda & 114 & 53,8 \\
\hline Controle & & \\
\hline Baixo Controle & 138 & 65,1 \\
\hline Alto Controle & 74 & 34,9 \\
\hline Apoio Social & & \\
\hline Baixo Apoio Social & 71 & 33,6 \\
\hline Alto Apoio Social & 140 & 66,4 \\
\hline Total & 212 & 100
\end{tabular}

*Diferenças dos $\mathrm{n}$ totais devem-se a perdas para algumas variáveis.

Os dados demonstram que o maior número de trabalhadores está na categoria de alta exigência, que combina alta demanda e baixo controle, $78(36,8 \%)$, seguido pelo trabalho passivo (baixa demanda e baixo controle) com 60 (28,3\%) (Tabela 4$)$.

Tabela 4 - Caracterização do tipo de exigência do trabalho no Modelo Demanda-Controle dos Agentes Comunitários de Saúde. Juiz de Fora, MG, Brasil, 2016

\begin{tabular}{lcc} 
Tipo de exigência no Trabalho & $\mathbf{n}$ & $\mathbf{\%}$ \\
\hline Trabalho de Baixa Exigência & 38 & 17,9 \\
\hline Trabalho Ativo & 36 & 17 \\
\hline Trabalho Passivo & 60 & 28,3 \\
\hline Trabalho de Alta Exigência & 78 & 36,8 \\
\hline Total & 212 & 100
\end{tabular}


O perfil feminino e de adultos jovens em plena fase produtiva da vida relaciona-se com o papel de cuidador destinado à mulher na sociedade brasileira e com o crescente número de mulheres assumindo papéis no mercado de trabalho. ${ }^{(12-13)}$

A inserção da mulher na ocupação dos postos de trabalho se deve às diversas transformações sociais ocorridas e por possuírem qualidades atribuídas ao sexo feminino, como cuidado e resistência. (14) Tal situação, no entanto, pode gerar vulnerabilidade, pois as tornam susceptíveis à discriminação de gênero e distribuição injusta de tarefas. Isso pode levá-las ao desenvolvimento de relações de trabalho marcadas por desigualdades, favorecendo desgastes e problemas de dimensões biológica e psicossocial. ${ }^{(15)}$

Da mesma forma que esses resultados, outros estudos também referem à feminização no trabalho dos ACSs e evidenciam o perfil majoritariamente do sexo feminino e faixa etária entre 20 e 50 anos de idade. ${ }^{(16-17)}$

Em relação à qualificação dos ACS, eles referem formação acima da exigida pela legislação para o exercício de sua função e, além disso, cabe ressaltar que atualmente existem diversos cursos que ofertam a formação técnica de ACS. ${ }^{(18)}$

Este perfil de escolaridade pode ser discutido, tendo em vista a complexidade de suas atividades no contexto de saúde, o que indica a necessidade de busca de qualificação e aprimoramento dos conhecimentos, para que possam executar as habilidades necessárias, reforçando as orientações à comunidade e assim desenvolver seu papel social de forma eficiente e eficaz. ${ }^{(19)}$

Ainda a este respeito, e considerando a literatura e um estudo sobre conhecimento em saúde bucal de ACS, destaca-se que $64,2 \%$ dos ACSs possuíam ensino médio completo e $26 \%$ referiram ter concluído ou estarem cursando o ensino superior, sendo que os cursos escolhidos em $15 \%$ dos casos eram cursos da saúde. ${ }^{(19)}$ A escolarização tem um valor importante e é socialmente reconhecida como meio de elevar-se social e profissionalmente, o que pode ser um anseio dos ACSs. Estes pertencem a camadas menos favorecidas e se sentem desprovidos, em diversos momentos de suas vidas, de recursos econômicos e vislumbram por meio da escolarização uma possibilidade de alcançar melhores condições de vida e de trabalho.

Quanto às características laborais, a população estudada está predominantemente trabalhando com carga horária dentro do limite constitucional, tendo em vista o proposto pela Constituição Federal de 1988 para que os trabalhadores não excedam 44 horas semanais. ${ }^{(20)}$ No entanto, ainda há um percentual que refere carga horária semanal total de todos os vínculos de trabalho maior que 40 horas, o que pode estar relacionado ao fato da maioria dos ACSs serem mulheres e possuírem dupla ou tripla jornada de trabalho, pois, além da carga horária de trabalho formal, realizam atividades voltadas ao cuidado da casa, família e filhos com turnos extra de trabalho. ${ }^{(14,21)}$

As mulheres inseridas no trabalho formal não estão isentas das atividades e responsabilidades do trabalho doméstico e cuidados com familiares. Portanto podem estar constantemente expostas a maiores cargas de trabalho, que podem gerar processos de desgastes biopsíquicos que levam ao sofrimento e estresse..$^{(4,14,21)}$

Destaca-se, pois, a importância da limitação da jornada de trabalho para os indivíduos, em diversos aspectos como biológico, social e econômico, visando poupar os trabalhadores de estressores organizacionais e de problemas decorrentes da fadiga e possibilitar vida social e ambiente familiar saudável. ${ }^{(20)}$

Dentre os estressores no trabalho de maior incidência estão: prolongadas jornadas de trabalho, trabalho noturno, e duplas ou triplas jornadas de trabalho. Estes afetam diretamente a saúde e o bemestar dos trabalhadores com impacto no comprometimento e satisfação no trabalho, o que implica na sua qualidade de vida. ${ }^{(20,22)}$

Em relação ao ACS, tal situação se torna peculiar, tendo em vista as atividades a ele designadas. Seu trabalho é comprometido por diversos fatores, pois é realizado no ambiente externo, o que 
proporciona maior exposição a riscos físicos, biológicos, mecânicos e psicossociais. Suas condições de trabalho são instáveis, uma vez que enfrenta dificuldades estruturais, exposição a fatores ambientais e climáticos e a doenças; condições salariais inadequadas; inexistência de limite entre o local de trabalho e sua moradia. Estas situações podem causar sobrecarga física e mental nas relações sociais e na saúde desse trabalhador. ${ }^{(7,23)}$

A característica tempo de serviço na APS se mostrou similar a outros estudos, caracterizando-se pelo predomínio de trabalhadores com mais de cinco anos de trabalho. Um estudo relacionado à percepção de estresse de servidores da atenção básica destaca uma média de 10,7 anos trabalhados na área de atuação. ${ }^{(24)}$ Tais proporções sugerem que há uma tendência de redução na rotatividade dos trabalhadores de saúde na Atenção Primária à Saúde, destacando, desta forma, maior quantitativo de profissionais com mais tempo de atuação na ESF.

No entanto, o tempo de trabalho pode favorecer o acúmulo de fatores estressantes, fazendo com que os trabalhadores não consigam superar as demandas e desenvolvam o estresse psicossocial. Além disso, deve-se levar em consideração que estes trabalhadores iniciaram suas atividades laborais muito novos, esta longa exposição pode acarretar em desequilíbrios físico e emocional do profissional, e desencadear dificuldades para lidar com as situações do cotidiano em seu ambiente de trabalho e impactar na sua saúde. ${ }^{(22)}$

A complexidade dos problemas de saúde decorrentes da exposição a fatores de riscos gerados pelo trabalho e a situação de vulnerabilidade social agregam exigências às equipes e consequentemente aos profissionais. Estes, muitas vezes, não estão preparados para resolver a maioria dessas questões, o que gera maior dificuldade e um cuidado diferenciado na realização das situações de rotina relacionadas aos processos saúde-doença da população e dos profissionais. ${ }^{(25)}$

O pressuposto de Karasek estabelece que o trabalho realizado em algumas condições possa propiciar o aparecimento de estresse psicossocial no trabalho e desencadear um processo de doença e sofrimento. Dados da Organização Mundial da Saúde indicam que mais de $90 \%$ da população mundial é afetada pelo estresse na atualidade, por viver em constantes exigências de atualização e necessidade de lidar com as transformações e se adaptar. ${ }^{(26)}$

Quanto aos tipos de trabalho e estresse laboral, os ACSs encontram-se predominantemente no trabalho passivo e de alta exigência. O trabalho de alta exigência é considerado como o grupo de exposição simultânea a elevadas demandas psicológicas e escasso controle sobre o processo de trabalho e o trabalho passivo é a segunda exposição mais problemática para a saúde dos trabalhadores. Segundo Karasek, esta categoria não favorece o desenvolvimento dos indivíduos e pode ser fator desestimulador e de desgaste, o que contribui para a insatisfação nos ambientes laborais. Essas condições podem favorecer o acometimento de reações adversas e a desgastes psicológicos levando os trabalhadores desenvolverem o estresse psicossocial no trabalho. ${ }^{(8-10)}$

A alta exigência e o baixo controle no trabalho geram desequilíbrios laborais e produzem aumento na liberação de hormônios do estresse nos indivíduos, que, por sua vez, conduzem os trabalhadores ao processo de adoecimento físico como lesões em coluna ou membros e psicológico como ansiedade, depressão e estresse. Diante do pouco controle e desgaste psicológico, os trabalhadores não terão condições para responder de forma adequada às circunstâncias ambientais. ${ }^{(9,10,27)}$

Pesquisas evidenciam que situações envolvendo baixo controle sobre o trabalho, como o que ocorre nos trabalhos de alta exigência e passivo, repercutem na falta de desafios. Isso favorece o aumento de riscos à saúde dos trabalhadores envolvidos e consequentemente redução do interesse e participação, insatisfação, habilidades e capacidade de tomar decisões e soluções no enfrentamento dos problemas laborais. ${ }^{(9,27)}$

No âmbito internacional, um estudo chileno que teve como objetivo analisar sintomas depressivos e sofrimento no trabalho identificou condições diferenciais para homens e mulheres, em que as mulheres têm significativa relação de interferência no trabalho e família em comparação com os homens. Utilizou-se o modelo de Karasek e obteve-se prevalência de exposição ao trabalho de risco psicossocial, sendo 16\% em trabalho de alta exigência e 10\% em estresse psíquico com baixo suporte social. No estudo, as mulheres se apresentaram com maior quantitativo em exposição a fatores de risco psicossocial no trabalho. Os autores ressaltam que esses dados têm sido corroborados por pesquisas 
internacionais em que os sintomas depressivos são evidenciados com prevalência significativamente mais elevada em mulheres do que em homens. ${ }^{(28)}$

Os transtornos mentais e comportamentais não parecem diferir entre os sexos, no entanto, segundo a OMS os transtornos depressivos representam o terceiro problema de saúde entre as mulheres nos países desenvolvidos e o quinto nos países subdesenvolvidos. Destaca-se, portanto, a prevalência dos transtornos de ansiedade e depressão no sexo feminino e também fatores psicológicos e sociais que podem interferir de forma significativa na saúde. O papel que as mulheres desempenham na sociedade e suas duplas ou triplas jornadas de trabalho as fazem se deparar com maiores estressores. ${ }^{(14,21)}$

Portanto, os elementos que compõem o ambiente de trabalho possuem riscos psicossociais e podem comprometer a saúde e qualidade de vida dos trabalhadores. Esses aspectos são variados e numerosos, interagem entre si e repercutem sobre a saúde física e psíquica dos indivíduos em seus ambientes laborais. ${ }^{(27)} \mathrm{Na}$ atenção à saúde, os serviços são marcados por relações psicossociais paradoxais, tendo em vista que a prestação do cuidado ao indivíduo pode gerar benefícios ao trabalhador, mas as relações desenvolvidas também podem ter efeito prejudicial à saúde deste. ${ }^{(1)}$

O estudo das condições de trabalho torna-se importante, uma vez que se, por um lado, o trabalho traz a possibilidade de realização, de relacionamento com os outros e de satisfação das necessidades básicas, por outro lado, ele pode ser responsável pelo adoecimento e desgaste ${ }^{(29)}$. O fato de a população apresentar alto apoio social, mesmo com as maiores proporções dos trabalhos prejudiciais à saúde, pode ser explicado através das características individuais de cada trabalhador, tendo em vista suas expectativas, realização pessoal e crenças em relação à recompensa e reconhecimento oferecido pela instituição em que trabalha. ${ }^{(30)}$

\section{- CONCLUSÃo}

O entendimento do trabalho desenvolvido pelos profissionais promove sensibilização e reflexão sobre os agravos à saúde mental do trabalhador da atenção primária. As características do trabalho do ACS reforçam a necessidade de adoção de medidas de promoção da saúde para proporcionar melhorias na sua rotina e no seu ambiente de trabalho, com o objetivo de minimizar os riscos e danos decorrentes das suas condições de trabalho.

Ressalta-se a importância do reforço e manutenção do apoio social por parte dos gestores e dos colaboradores, pois a presença de alto apoio social pode agir de modo a minimizar ou proteger os trabalhadores dos efeitos negativos advindos da exposição a agentes estressores.

\section{REFERÊNCIAS}

1. Santos TM, Camponogara S. Um olhar sobre o trabalho de enfermagem e a ergologia. Trab. educ. saúde [Internet]. 2014 [acesso em 2017 maio 10]; 12(1). Disponível em: http://dx.doi.org/10.1590/S1981-77462014000100009.

2. Kogien M, Cedaro JJ. Public emergency department: the psychosocial impact on the physical domain of quality of life of nursing professionals. Rev. Latino-Am. Enfermagem [Internet]. 2014 [acesso em 2017 abr 24]; 2(1). Disponível em: http://dx.doi.org/10.1590/0104-1169.3171.2387.

3. Seligmann-Silva E, Bernardo MH, Maeno M, Kato M. Saúde do Trabalhador no início do século XXI. Rev. bras. saúde ocup. [Internet]. 2010 [acesso em 2017 abr 24]; 35(122). Disponível em: http://dx.doi.org/10.1590/S030376572010000200001.

4. Karino ME, Felli VEA, Sarquis LMM, Santana LL, Silva SR, Teixeira RC. Cargas de trabalho e desgastes dos trabalhadores de enfermagem de um Hospital-escola. Ciênc. cuid. saúde [Internet]. 2015 [acesso em 2016 mar 26]; 14(2). Disponível em: http://dx.doi.org/10.4025/cienccuidsaude.v14i2.21603.

5. Santana LL, Miranda FM D'A, Karino ME, Baptista PCP, Felli VEA, Sarquis LMM. Cargas e desgastes de trabalho vivenciados entre trabalhadores de saúde em um hospital de ensino. Rev. Gaúcha Enferm. [Internet]. 2013 [acesso em 2016 mar 20]; 34(1). Disponível em: http://dx.doi.org/10.1590/S1983-14472013000100008. 
6. Martins LF, Laport TJ, Menezes VP, Medeiros PB, Ronzani TM. Esgotamento entre profissionais da Atenção Primária à Saúde. Cienc. saude colet. [Internet]. 2014 [acesso em 2016 mar 20]; 19(12). Disponível em: http:// dx.doi.org/10.1590/1413-812320141912.03202013.

7. Gomes MF, Lima ASR, Feitosa LS, Netto VBP, do Nascimento RD, Andrade MS. Riscos e agravos ocupacionais: percepções dos agentes comunitários de saúde. J. res.: fundam. care [Internet]. 2015 [acesso em 2016 mar 20]; 7(4). Disponível em: http://dx.doi.org/10.9789/2175-5361.2015.v7i4.3574-3586.

8. Alves MGM, Chor D, Faerstein E, Lopes CS, Werneck GL. Versão resumida da "job stress scale": adaptação para o português. Rev. Saude Públ. [Internet]. 2004 [acesso em 2016 mar 20]; 38(2). Disponível em: http://dx.doi. org/10.1590/S0034-89102004000200003.

9. Karasek R, Theorell T. Healthy work: stress, productivity and the reconstruction of working life. New York: Basic Books; 1990.

10. Alves MGM, Braga VM, Faerstein E, Lopes CS, Junger W. The demand-control model for job strain: a commentary on different ways to operationalize the exposure variable. Cad. saúde publ. [Internet]. 2015 [acesso em 2016 abr 10]; 31(1). Disponível em: http://dx.doi.org/10.1590/0102-311X00080714.

11. Ministério da Saúde (BR). Conselho Nacional de Saúde. Diretrizes e normas regulamentadoras de pesquisa envolvendo seres humanos. Resolução n. 466, de 12 de dezembro de 2012. Brasília; 2012.

12. Baptistini RA, Figueiredo TAM. Agente comunitário de saúde: desafios do trabalho na zona rural. Ambient. soc. [Internet]. 2014 [acesso em 2016 abr 21]; 17(2). Disponível em: http://dx.doi.org/10.1590/S1414-753X2014000200005.

13. Galavote HS, Prado TN, Maciel ELN, Lima RCD. Desvendando os processos de trabalho do agente comunitário de saúde nos cenários revelados na Estratégia Saúde da Família no município de Vitória (ES, Brasil). Cienc. saude colet. [Internet]. 2011 [acesso em 2016 abr 21]; 16(1). Disponível em: http://dx.doi.org/10.1590/S141381232011000100026.

14. Barbosa RHS, Menezes CAF, David HMSL, Bornstein VJ. Gênero e trabalho em Saúde: um olhar crítico sobre o trabalho de agentes comunitárias/os de Saúde. Interface (Botucatu) [Internet]. 2012 [acesso em 2016 abr 10]; 16(42). Disponível em: http://dx.doi.org/10.1590/S1414-32832012000300013.

15. Landsbergis PA, Dobson M, Koutsouras G, Schnall P. Job Strain and Ambulatory Blood Pressure: A MetaAnalysis and Systematic Review. Am J Public Health [Internet]. 2013 [acesso em 2016 abr 10]; 103(3). Disponível em: http://dx.doi.org/10.2105/AJPH.2012.301153.

16. Melo MB, Quintão AF, Carmo RF. O Programa de Qualificação e Desenvolvimento do Agente Comunitário de Saúde na perspectiva dos diversos sujeitos envolvidos na atenção primária em saúde. Saude Soc. [Internet]. 2015 [acesso em 2016 abr 10]; 24(1). Disponível em: http://dx.doi.org/10.1590/S0104-12902015000100007.

17. Menegussi JM, Ogata MN, Rosalini MHP. O agente comunitário de saúde como morador, trabalhador e usuário em São Carlos, São Paulo. Trab. educ. saúde [Internet]. 2014 [acesso em 2016 mar 10]; 12(1) Disponível em: http://dx.doi.org/10.1590/S1981-77462014000100006.

18. Brasil. Lei n.11.350, de 5 de outubro de 2006. Revoga a Lei n.0.507, de 10 de julho de 2002. Cria a profissão de Agente Comunitário de Saúde e dá outras providências. Diário Oficial da União, Brasília, 6 out. 2006.

19. Gouvêa GR, Silva MAV, Pereira AC, Mialhe FL, Cortellazzi KL, Guerra LM. Evaluation of knowledge of Oral Health of Community Health Agents connected with the Family Health Strategy. Cienc. saude colet. [Internet]. 2015 [acesso em 2016 mar 10]; 20(4). Disponível em: http://dx.doi.org/10.1590/1413-81232015204.00682014.

20. Furlan Junior PF. A redução da jornada de trabalho e seus benefícios. Revista Eletrônica do CEMOP [Internet]. 2012 [acesso em 10 mar 2016]; (02). Disponível em: http://www.memoriaoperaria.org.br/revistaeletronica/paulo_ furlan.pdf

21. Vasconcelos SP, Fischer FM, Reis AOA, Moreno CRC. Fatores associados à capacidade para o trabalho e percepção de fadiga em trabalhadores de enfermagem da Amazônia ocidental. Rev. bras. epidemiol. [Internet]. 2011 [acesso em 2016 mar 20]; 14(4). Disponível em: http://dx.doi.org/10.1590/S1415-790X2011000400015.

22. Goulart Junior E, Cardoso HF, Domingues LC, Green RM, Lima TR. Trabalho e Estresse: Identificação dos Estressores Ocupacionais em Trabalhadores de uma Unidade Administrativa de uma Instituição Pública de 
Ensino Superior (IES). Revista GUAL [Internet]. 2014 [acesso em 2016 mar 20]; 7(1). Disponível em: https://doi. org/10.5007/1983-4535.2014v7n1p1.

23. Mascarenhas $\mathrm{CHM}$, Prado FO, Fernandes MH. Fatores associados à qualidade de vida de Agentes Comunitários de Saúde. Cienc. saúde colet. [Internet]. 2013 [acesso em 2016 mar 20]; 18(5). Disponível em: http://dx.doi. org/10.1590/S1413-81232013000500023.

24. Silva MG, Barros BP. Percepção de estresse de servidores na Atenção Básica de Saúde de Dourados-Ms. Saúde em Redes [Internet]. 2015 [acesso em 2016 mar 20]; 1(4). Disponível em: http://dx.doi.org/10.18310/24464813.2015v1n4p35-52.

25. Silva TL, Dias EC, Pessoa VM, Fernandes LMM, Gomes EM. Saúde do trabalhador na Atenção Primária: percepções e práticas de equipes de Saúde da Família. Interface (Botucatu) [Internet]. 2014 [acesso em 2016 mar 20]; 18(49). Disponível em: https://dx.doi.org/10.1590/1807-57622013.0227.

26. Bezerra FN, Silva TM, Ramos VP. Occupational stress of nurses in emergency care: an integrative review of the literature. Acta Paul. Enferm. [Internet]. 2012 [acesso em 2016 mar 20]; 25(n.esp2):151-156. Disponível em: http:// dx.doi.org/10.1590/S0103-21002012000900024.

27. Magnago TSBS, Lisboa MTL, Griep RH, Zeitoune RCG, Tavares JP. Condições de trabalho de profissionais da enfermagem: avaliação baseada no modelo demanda-controle. Acta Paul. Enferm. [Internet]. 2010 [acesso em 2016 mar 20]; 23(6). Disponível em: http://dx.doi.org/10.1590/S0103-21002010000600015.

28. Ansoleaga E, Vézina $M$, Montaño R. Síntomas depresivos y distrés laboral en trabajadores chilenos: condiciones diferenciales para hombres y mujeres. Cad. saúde publ. [Internet] 2014 [acesso em 2016 mar 20]; 30(1). Disponível em: http://dx.doi.org/10.1590/0102-311X00176912.

29. Oliveira, MP, Kusumota L, Marques S, Ribeiro RCHM, Rodrigues RAP, Haas VJ. Trabalho e qualidade de vida relacionada à saúde de pacientes em diálise peritoneal. Acta Paul. Enferm. [Internet]. (2012) [acesso em 2016 mar 20]; 25(3). Disponível em: https://dx.doi.org/10.1590/S0103-21002012000300006.

30. Fonseca ISS, Moura SB. Apoio social, saúde e trabalho: uma breve revisão. México. Psicol. Am. Lat. [Internet]. 2008 [acesso em 2016 mar 19]; (15). Disponível em: http://pepsic.bvsalud.org/scielo.php?script=sci_ arttext\&pid=S1870-350X2008000400012. 